\title{
Artificial Intelligence Teaches Drugs to Target Proteins by Tackling the Induced Folding Problem
}

\author{
Ariel Fernández*
}

Cite This: Mol. Pharmaceutics 2020, 17, 2761-2767

Read Online

ACCESS | Llll Metrics \& More | 回 Article Recommendations

ABSTRACT: We explore the possibility of a deep learning (DL) platform that
steers drug design to target proteins by inducing binding-competent
conformations. We deal with the fact that target proteins are usually not fixed
targets but structurally adapt to the ligand in ways that need to be predicted to
enable pharmaceutical discovery. In contrast with protein folding predictors, the
proposed DL system integrates signals for structural disorder to predict
conformations in floppy regions of the target protein that rely on associations
with the purposely designed drug to maintain their structural integrity. This is
tantamount to solve the drug-induced folding problem within an AI-empowered
drug discovery platform. Preliminary testing of the proposed DL platform reveals
that it is possible to infer the induced folding ensemble from which a
therapeutically targetable conformation gets selected by DL-instructed drug design.

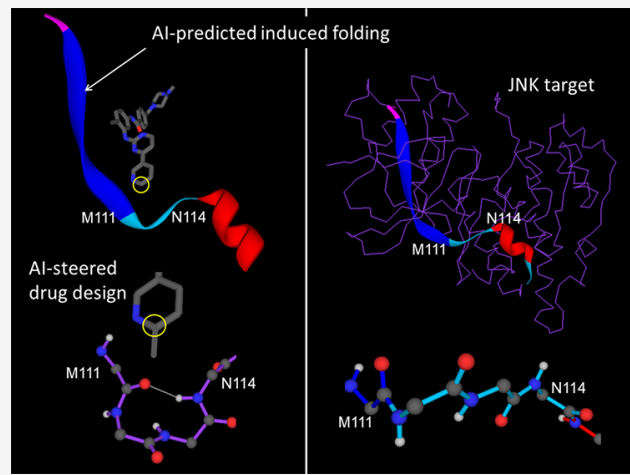

KEYWORDS: drug design, molecular targeted therapy, artificial intelligence, deep learning, induced protein folding

\section{STEERING DRUGS TO TARGET PROTEINS BY LEARNING TO RECOGNIZE BINDING-INDUCED FOLDS}

The implementation of deep learning (DL) methods enabled remarkable progress toward solving the protein folding problem, ${ }^{1}$ that is, the prediction of the $3 \mathrm{D}$ structure adopted by a protein chain based on its amino acid sequence. ${ }^{2}$ It is broadly acknowledged that this challenge has been met with considerable success by a machine learning system named AlphaFold $^{1}$ created by Deep Mind Technologies. Here we explore the possibility to adapt this platform for a different set of needs associated with drug design. We effectively deal with the fact that target proteins are typically not fixed targets: they structurally adapt to the ligand in ways that need to be predicted to empower pharmaceutical discovery. Specifically, we aim at predicting structures that only prevail within obligatory complexes, that is, structures for nonautonomous folders. The goal is to instruct drug design to target specific conformations predicted to be reliant on association with the purposely designed drug to maintain their structural integrity. This is tantamount to adapt the AlphaFold platform to solve the druginduced folding problem.

To adapt the AlphaFold platform, we first review its architecture and associated workflow. AlphaFold trains a multilayered convolutional neural network $(\mathrm{CNN})^{3}$ with PDB-reported protein structures and generates 3D models that fit accurate predictions of distances between residue pairs. Such predictions are based on a 2D-array representation of pairwise coevolutionary information, ${ }^{4}$ under the premise that if the evolution of two residues is correlated within a multiple sequence alignment, it is likely that they are spatially related. The evolutionary information is concatenated with residue profiling including amino acid identity and secondary structure prediction. Local propagation of distance constraints from one layer to the next is achieved via progressively dilated convolutional operations, ${ }^{5}$ where convolutional "filters" allow incorporation of features not just from neighbors of a pixel but also from further afield, incorporating surrounding context by expanding the receptive field of the filter. In this way, AlphaFold fulfills a major imperative since the elucidation of protein structure is key to delineate biological function, and its experimental determination often eludes available methods. ${ }^{1}$

Protein structure prediction assumes that the chain will adopt a $3 \mathrm{D}$ structure and that the structure is unique. ${ }^{1,2}$ In practice, many proteins cannot fold autonomously, and the structure is not unique but dependent on binding partnerships, reliant on such associations to maintain its integrity. ${ }^{6}$ In fact, more often than not, the binding context selects the protein fold from within an ensemble of closely related folding possibilities that we hereby name the induced folding ensemble (IFE). For example, proteins such as transcription factors typically possess only

Received: April 29, 2020

Revised: June 15, 2020

Accepted: June 17, 2020

Published: June 17, 2020 


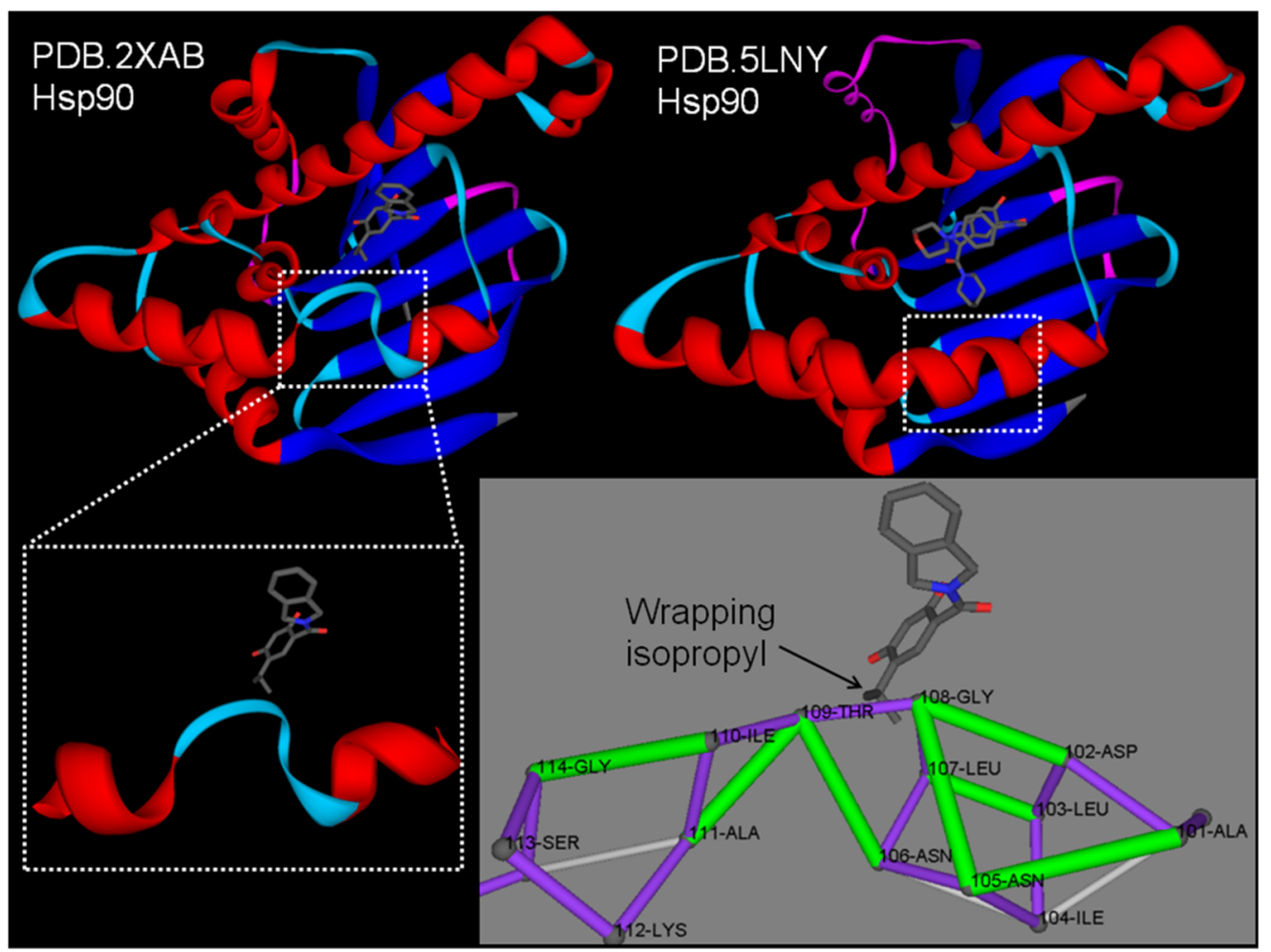

Figure 1. Conformational diversity of "holo forms" for cancer target Hsp90 induced by different drug ligands resulting from the intrinsic disorder propensity of a region in the protein, as the region is reliant on binding partnerships to maintain structural integrity. The $100-120$ helix in Hsp90 gets distorted, developing a kink as the ligand in the complex PDB.5LNY gets replaced for that in PDB.2XAB. The helical region has a disorder propensity, which translates into susceptibility to fold into a binding-induced conformation that varies according to the ligand. The ligand in PDB.2XAB has a protruding isopropyl group capable of exogenously contributing to the wrapping of the dehydron-rich helix kink, specifically wrapping the dehydrons T109-A111 and N106-T109. In this way, the ligand determines or selects the binding-competent-induced folding. The protein chain is shown simplified as a polygonal of virtual bonds (purple) joining the $\alpha$-carbons of contiguous residues along the chain. BHBs are indicated as lines joining the $\alpha$-carbons of paired residues, with well-wrapped bonds marked in gray and dehydrons in green. For the computation of BHB wrapping and identification of dehydrons, the reader may consult other work. ${ }^{12-14}$

induced structures; that is, their fold cannot prevail in an "apo" (separated) form, ${ }^{7}$ and antibodies usually present antigeninduced conformational multiplicity, ${ }^{8}$ while proteins which constitute drug targets often have a variety of "holo" (withincomplex) forms, depending on the ligand/drug they associate with. $^{9}$

The latter context is the focus of this work and may be illustrated by the structural diversity of the stress-responsive molecular chaperone Hsp90, ${ }^{10}$ a cancer target that adopts different structures depending on the drug-target complex (Figure 1). This type of induced folding diversity should prompt rational drug designers to focus on inferring the target's IFE, rather than a unique $3 \mathrm{D}$ structure, probably only suitable for autonomous folders, ${ }^{11}$ forming nonobligatory complexes. Thus, efforts in structure-based drug design are often marred by the need to encompass the target structural adaptation., Obviously, the IFE concept shifts the focus of structure prediction, especially of DL prediction, that must be adapted to infer a priori holo forms, introducing significant complications. Here we propose a DL scheme to enable IFE prediction of pharmaceutically relevant holo forms, geared at tackling the "drug-induced folding problem", possibly the next frontier for a rational drug designer involved in lead optimization.

\section{NETWORK ARCHITECTURE FOR DEEP LEARNING} TO INDUCE DRUG-TARGETABLE PROTEIN FOLDS

Induced folding implies that the protein relies on binding partnerships to maintain structural integrity ${ }^{6-9}$ with the ligand selecting a conformation from within the IFE. Thus, to delineate a suitable CNN architecture of a DL-based predictor of the IFE requires that we first identify the culprit for the loss of structural integrity. Since backbone hydrogen bonds (BHBs) are determinants of protein structure, it is obvious that hydration of backbone amides and carbonyls competes with intramolecular hydrogen bond formation, exerting a structuredisruptive effect. An autonomous folder ${ }^{11}$ is thus capable by itself of shielding most BHBs from the disruptive effects of backbone hydration. Thus, protein integrity may be said to be contingent on the structure, by itself or within a complex, being capable of preventing backbone hydration. This leads us to propose a descriptor of the degree of autonomy of the protein fold (or its degree of reliance on binding partnerships), which we named "wrapping" ${ }^{12,13}$ An underwrapped BHB constitutes a structural defect, known as dehydron, ${ }^{13}$ possessing an insufficient number of side-chain nonpolar groups clustered around the $\mathrm{BHB}$, so that dehydrons are exposed to structuredisruptive hydration. ${ }^{6,7,12,13}$ An extended underwrapped region (dehydron cluster) has a high propensity to be disordered ${ }^{7}$ in the apo form, since backbone hydration is likely to prevail over structure formation. Thus, induced folding may be interpreted as the optimal structural adaptation to a binding ligand to ensure 
the maximum shielding of underwrapped regions, thereby contributing to the stabilization of a specific holo form.

Dehydrons and wrapping may be computed from structural coordinates of proteins, $6,7,12,13$ and the computation may be incorporated in the feature-extraction workflow of the DL platform. ${ }^{14}$ To that end, we introduce the extent of hydrogenbond wrapping, $\mathrm{w}$, indicative of the number of side-chain nonpolar groups contained within a "desolvation domain" that defines the BHB microenvironment in a training reported structure. Being marginally wrapped, dehydrons are located in the tail of the distribution of $\mathrm{w}$-values across BHBs in the structural database. The dehydrons in the protein structure compromise its integrity and promote protein associations as a means to exclude surrounding water. In this way, dehydrons become determinants for protein association because, by decreasing charge screening, exogenous removal of water from the dehydron microenvironment strengthens and stabilizes the electrostatic interaction that underlies the BHB. Ample bioinformatics evidence on the distribution of dehydrons at interfaces of protein complexes supports this picture, ${ }^{13}$ pointing to dehydrons as key factors driving complex formation, with dehydron-rich regions being flexible and hence susceptible of undergoing binding-induced folding. ${ }^{9}$ To illustrate this picture, the Hsp90 helical 101-128 region in the complex reported in PDB entry 5LNY becomes disrupted when Hsp90 forms a different complex (PDB.2XAB), as shown in Figure 1. The structural difference arises as a kink forms with dehydrons N106T109 and T109-A111, which in turn get exogenously wrapped by the protruding isopropyl group in the drug inhibitor that binds Hsp90 in the complex reported in PDB.2XAB. As the example suggests, the $a$ priori inference of induced folding states would be essential for a rational drug designer, seeking to control drug affinity and specificity through lead optimization to select the holo form from within the IFE.

The previous discussion suggests that leveraging a DL platform to predict induced conformations hinges on a wrapping representation of the protein chain in the feature extraction phase. The structure wrapping must lend itself to a tensor representation wherein information is describable and processed on a multidimensional array (tensor) of digital entries within a layered architecture. Thus, the steering of the drug design process to target a binding-competent-induced fold involves the inference of the IFE using the primary sequence of the target protein as an input. This task requires an adaptation of the AlphaFold platform to incorporate the following elements:

(1) In contrast with AlphaFold and other structure predictors, residues need to be profiled incorporating an additional key signal: the sequence-based prediction of intrinsic disorder, a descriptor of the propensity of a window along the chain to be inherently unstructured as the protein is taken in isolation, i.e., as an autonomous folder. ${ }^{15,16}$ The disorder score ranges from 1 (certainty of disorder) to 0 (certainty of order). Regions predicted to be disordered are expected to be reliant on binding partnerships to improve the wrapping of $\mathrm{BHBs}$ to the point where they can be sustainable. ${ }^{6,9}$ Thus, the disorder score signal needs to be integrated into the residue profiling for a sequence-based inference of the IFE. A clear illustration is provided by the induced folding diversity in Hsp90: the predicted helicity of the region 100-120 (Figure 1) is partially overlapping with an overwriting signal of intrinsic disorder for the region $80-114$ (we follow the PDB numbering 16-224 of the Hsp90 chain). This signal was spotted in a sequence-based prediction of intrinsic disorder for Hsp90 regarded as an autonomous folder. The disorder plot was generated on January 30, 2020, at 19:31 by the Predictor of Native Disorder (PONDR) in its version XL1-XT, ${ }^{15}$ freely accessible from http:/ / www. pondr.com/.

This is a clear indication that the helix would be partially unsustainable if the protein is regarded as an autonomous folder and can be partially disrupted or distorted by induced folding depending on the ligand, as it is indeed the case in the overlapping region 100-114 (Figure 1). Thus, a kink is induced in the predicted helical region, whereby nonhelical dehydrons N106-T109 and T109-A111 are formed and become sustainable as they get wrapped exogenously by the isopropyl group in the Hsp90-binding ligand reported in PDB.2XAB.

(2) Residues paired by BHBs are represented within a triangular 2D-array (the information plotted is symmetric) inputted with pairwise coevolutionary information., The underlying premise is that, of the evolution of two residues correlates across a multiple sequence alignment, it is likely that they are spatially related. The array is concatenated with residue profiling in the same way as it is done in AlphaFold, except for the incorporation of the local disorder propensity as an overwriting signal.

(3) In contrast with AlphaFold, a third dimension is needed to identify the residues wrapping the BHBs that will emerge during the feature extraction phase of $\mathrm{DL} .^{13,14}$ Thus, wrapping components are identified upon the structural information within a $3 \mathrm{D}$-tensor whereby a matrix $\boldsymbol{Y}$, evolving from the evolutionary coupling matrix (ECM), is constructed to ultimately indicate residues $(i, j)$ paired by $\mathrm{BHBs}$, while the wrapping of the $\mathrm{BHB}$ is represented along $\boldsymbol{Y}$-orthogonal vector $\boldsymbol{z}_{i j}$ for entry $\boldsymbol{Y}_{i j}$. Thus, the DL flow operates on the tetrahedral $N \times N \times N$ tensor $Y \otimes Z=$ $\left[Y_{i j} \otimes z_{i j}\right]_{i j}(N=$ chain length).

(4) Feature extraction requires a dilated convolutional layered architecture ${ }^{1,3}$ where, in a generic layer denoted $\mathrm{F}$, the convolutional kernel becomes a $3 \times 3 \times 3$ tetrahedral tensor $(\boldsymbol{F})$ that evolves during the parameter optimization process turning into a filter. This filter extracts a structural pattern drawn from the training set in a curated PDB-derived database. ${ }^{14}$ The product of the convolutional operation $(\mathrm{Y} \otimes \mathrm{Z}) * \boldsymbol{F}$ is thus a tensor with entries indicating sums of entry-by-entry multiplication (Frobenius inner product) of $Y \otimes Z$ by tensor $F$ as the latter slides along $3 \times 3 \times 3$ tetrahedral sectors of $Y \otimes Z$ with stride 2 to produce the $(\mathrm{N}-2)^{3}$ convolved tensor $[\widehat{\mathbf{Y} \otimes \mathbf{Z}}]_{\mathrm{F}}=(\mathbf{Y} \otimes \mathbf{Z}) * \boldsymbol{F}$. Thus, large value entries in the convolved tensor are indicative of regions where the configuration more clearly resembles the kernel/filter. Within the F-layer, the convolved tensor $[\widehat{\mathbf{Y} \otimes \mathbf{Z}}]_{\mathrm{F}}$ is expanded into the "F-feature-discerning" $\mathrm{N}^{3}$-tensor $(Y \otimes Z)_{\mathrm{F}}$ by spanning the F-pattern for the largest value entries in $[\widehat{\mathbf{Y} \otimes \mathbf{Z}}]_{\mathrm{F}}$ while retaining the original entries from $Y \otimes Z$ for those $3 \times 3 \times 3$ tetrahedral sectors that do not yield a significant inner product. The F-filter is then dilated within an F-spanned multilayered architecture to incorporate the surrounding context by enlarging the receptive field size of the convolution kernel. In this way, we incorporate features not just from pixel neighbors but 
(a)

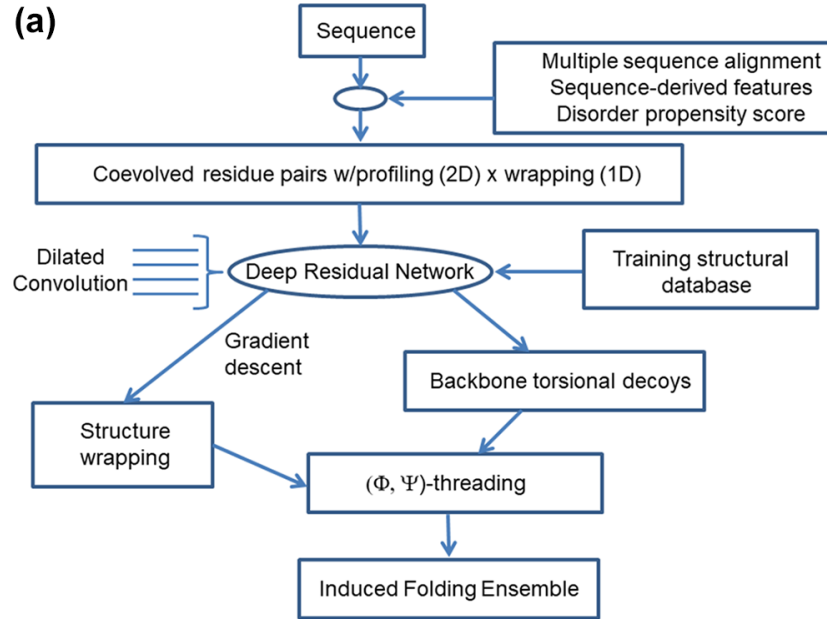

(c)

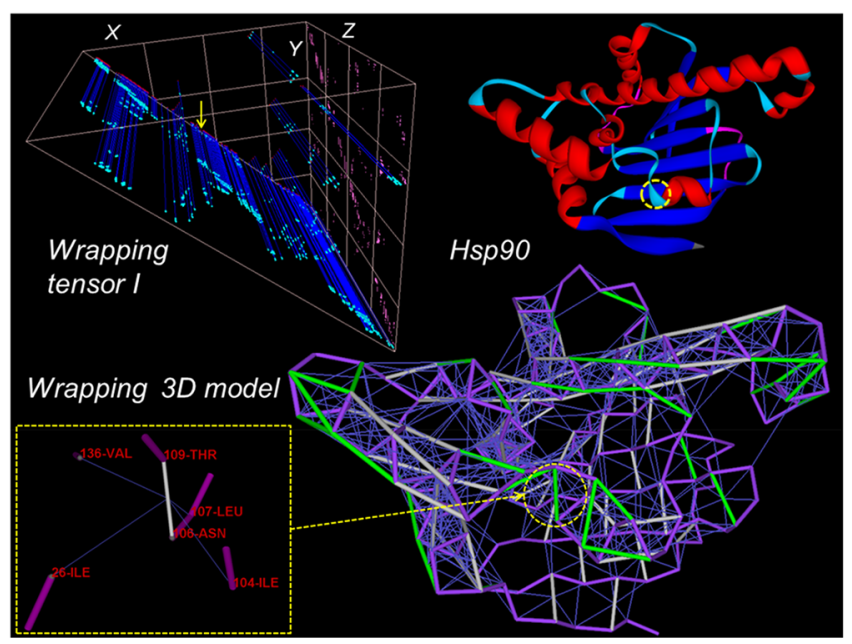

(b)
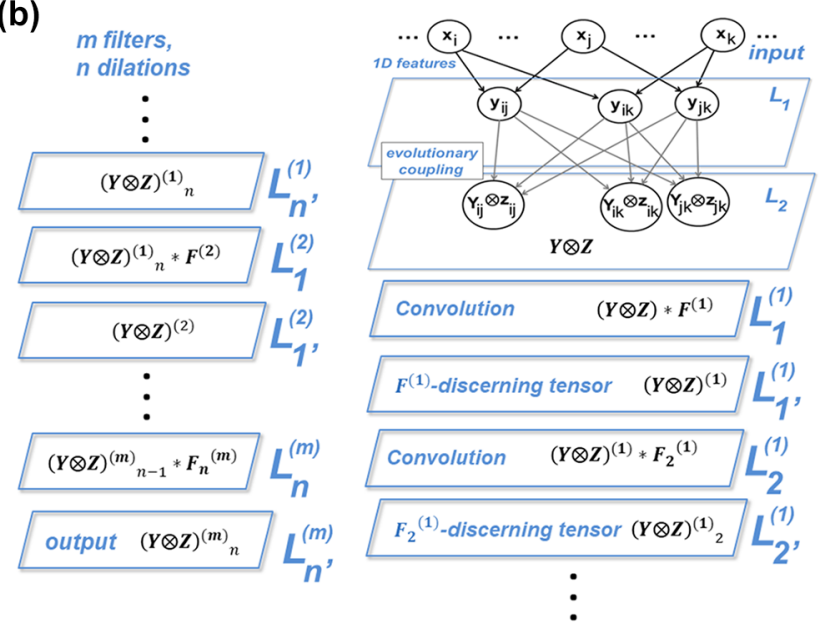

(d)

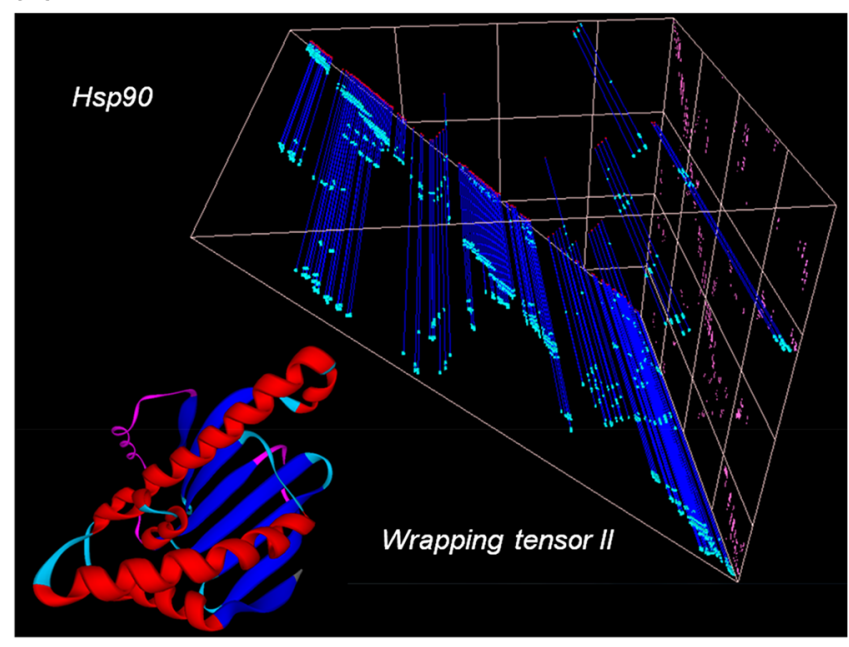

Figure 2. DL discovery platform and its inference of the IFE for Hsp90. (a) Workflow for the DL system. (b) Architecture of the dilated convolutional network required to infer structural wrapping patterns. The dummy indices $i, j, k$ indicate residues along the chain, and the $1 \mathrm{D}$ features, including disorder propensity, are subsumed vectorially in $\boldsymbol{x}_{i}, \boldsymbol{x}_{j}, \boldsymbol{x}_{k}, \ldots$; for feature extraction, the network contains mxnx 2 hidden layers labeled $\mathrm{L}^{(1)}{ }_{1}, \ldots, \mathrm{L}^{(m)}{ }_{n}(m=$ number of filters/convolution kernels, $n=$ number of dilations per filter). In the cases reported in this study, we used $m=120$ and $n=6$, so the network has 1440 hidden layers. (c) Component I of the Hsp90 IFE, specified by the tetrahedral wrapping tensor, the decoded 3D wrapping model, and the threaded 3D backbone conformation in ribbon rendering. The $X, Y, Z$ axes in the tensor indicate residue numbers $16-224$ along the chain (we respect $\mathrm{PDB}$ numbering for this contour variable). The inferred $\mathrm{BHBs}$ are represented in the triangle in the background of the tetrahedral tensor, while the residues contributing to the wrapping of each $\mathrm{BHB}$ are identified along the axis orthogonal to the background triangle. Thus, the wrapping residues are shown on the square on the right. The blue lines emanating from each BHB and orthogonal to the background triangle contain the residues (light blue boxes) wrapping the BHB. In the $3 \mathrm{D}$ wrapping model of the chain, a residue contributing to the wrapping of a BHB is indicated by a thin blue line between the $\alpha$-carbon of the wrapper and the center of the wrapped BHB. For further clarification, the 106-109 BHB is identified on the tensor (yellow arrow), wrapping 3D model, and structural rendering (yellow circles). This BHB is wrapped by residues 26, 104, 107, and 136, as depicted in the zooming of the wrapping 3D representation. (d) Component II of the IFE for Hsp90, specified by wrapping tensor and ribbon rendering of the 3D structure.

from further afield by creating $3 \times 3 \times 3$ tetrahedral filters with the pixels further and further apart. Thus, each neuron in a hidden layer only receives input from the local region of what the input to the layer is, and with the dilations, we enlarge the receptive field of the convolution kernel. So the hidden layer for filter $F$ is actually spanning a bunch of hidden layers where the dilated filters $F_{1}=F$, $F_{2}, F_{3}, \ldots$ are successively applied, with the subindex giving the value of the dilation coefficient.

(5) The training of the CNN enables optimization of the set of filters $\mathcal{F}=\left\{\mathrm{F}^{(1)}, \mathrm{F}_{2}^{(1)}, \ldots, \mathrm{F}^{(2)}, \mathrm{F}_{2}^{(2)}, \ldots\right\}$ by minimizing the sum of Frobenius norms ( $\|\cdot\|$, sum of squared entries) for tensors $Y \otimes Z-\overline{Y \otimes Z}$, where $\overline{Y \otimes Z}$ indicates the structure-based computed wrapping of each $\mathrm{BHB}$ in a PDB-reported structure $(\xi)$ picked from the training set $(\mathcal{T})$, and $Y \otimes Z$ is the DL-based inference of the structure wrapping obtained from the protein sequence of the PDBreported structure. Thus, the loss function $\mathcal{L}(\mathcal{F})$ for the DL network becomes

$$
\mathcal{L}(\mathcal{F})=\mid \mathcal{T}^{-1} \sum_{\xi \in \mathcal{T}}\|(\boldsymbol{Y} \otimes \boldsymbol{Z}-\overline{\boldsymbol{Y} \otimes \boldsymbol{Z}})(\xi)\|
$$

where $|\mathcal{T}|$ denotes the number of structures in the training set.

While the tensor representation of structure wrapping allows suitable processing in a DL platform with Tensor Flow, ${ }^{17}$ the tensor should get decoded into a $3 \mathrm{D}$ rendering specifying relative spatial locations of wrapping residues vis-à-vis the BHBs 


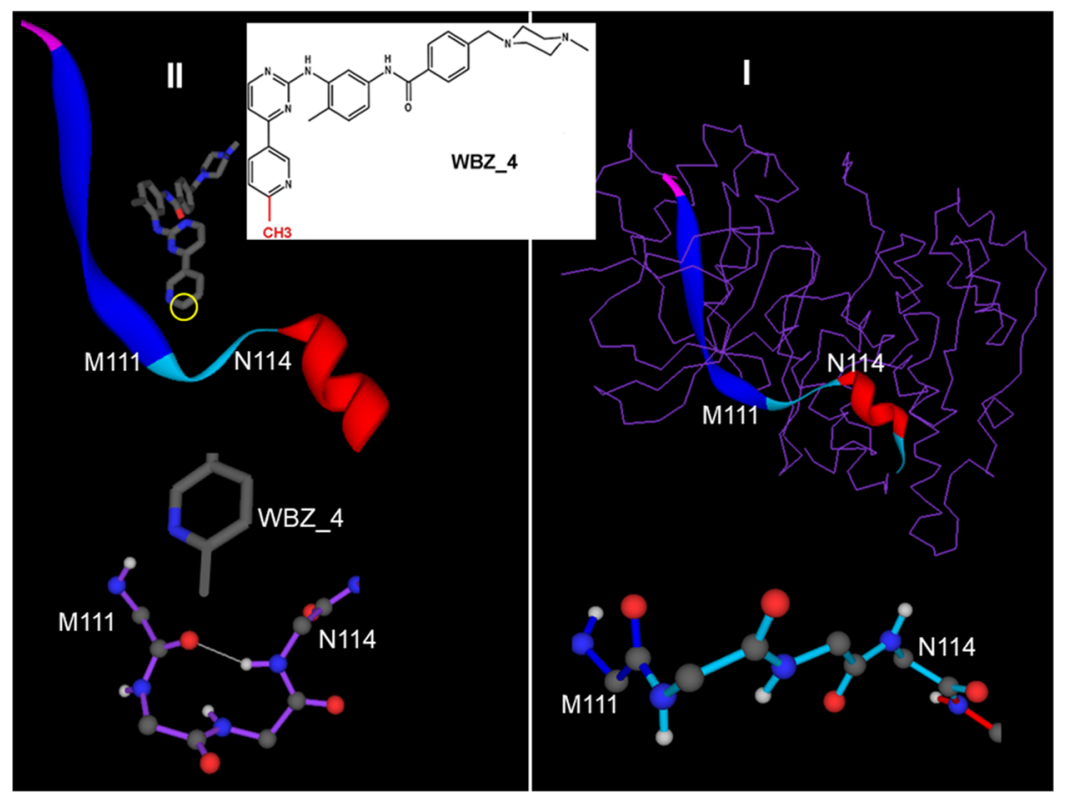

Figure 3. IFE inferred by the DL platform for JNK, a target of WBZ_4 and not of the parental drug imatinib. The apo form is component I, and the holo form is II. The BHB M111-N114 (actually a dehydron) is induced upon binding to WBZ_4 and absent in the apo form. The two components have identical structure except for the region where induced folding occurs, indicated by the ribbon rendering. This example illustrates the application of AI to steer drug design in order to target a specific protein by learning to induce a drug-binding-competent fold.

to enable the threading of structural decoys onto it (Figure $2 a-$ $c)$. The threading requires a separate inference of the torsional $(\Phi, \Psi)$-conformation of the protein backbone, as performed by AlphaFold, and a fitting of the backbone torsional model onto the decoded 3D model of structure wrapping to determine sidechain locations to the level of resolution where it becomes possible to decide whether they contribute to wrap-specific BHBs (Figure 2c). The full workflow of the DL platform is delineated in Figure 2a, while the network architecture is depicted in Figure $2 b$.

\section{DRUG DESIGN BY TEACHING TO INDUCE TARGETABLE FOLDS}

A DL prototype has been constructed as described in Figure 2a, following the premises $1-5$ given above. Details for a basic precursor of this system were previously reported. ${ }^{14}$ The precursor system has been modified (mutatis-mutandis) to infer induced folding possibilities. The repurposing requires the following: (a) The integration of a sequence-based prediction of the disorder ${ }^{15,16}$ as a signal overwriting secondary structure prediction in the residue profiling. This signal is essential to identify regions reliant on binding partnerships to maintain structural integrity, ${ }^{7,9}$ and hence susceptible to induced folding in consonance with the ligand. (b) The incorporation of dilated convolution in the layered architecture for feature extraction. This is essential to add nonlocal context to the prediction of induced folding, as the receptive field for each layer input gets progressively magnified in accord with the value of the dilation parameter for a given convolution kernel or filter during the feature extraction phase of the DL processing.

The IFE for cancer target Hsp90, obviously excluded from the training set and obtained by incorporating the prediction of intrinsic disorder is made up of two components, I, II, displayed in Figure $2 b, c$. The display includes the structure wrapping tensor and ribbon rendering of the $(\Phi, \Psi)$-threaded conformation. The RMSDs of backbone Cartesian coordinates for I and II relative to the structural coordinates within the complexes (PDB.2XAB and PDB.5LNY, respectively) are 1.9 and $1.2 \AA$.

\section{AI INSTRUCTS ON REWORKING A DRUG TO TARGET A PROTEIN}

Drug-based targeted therapy, aimed at blocking specific dysfunctional proteins, often faces a major obstacle due to induced folding, a hard-to-predict phenomenon that often generates unexpected and undesirable cross-reactivity, while making the intended target elusive to molecular recognition. ${ }^{9}$ We advocate that $\mathrm{DL}$ can steer drug design to achieve therapeutic impact by controlling the induced folding in the target protein. The way DL may teach the drug to target the protein is apparent as we focus on reworking the anticancer drug imatinib $^{18}$ into the prototype WBZ_4 ${ }^{9,13}$ (Figure 3). Exploiting wrapping differences among proteins that share a common fold as a filter for selectivity, a chemical modification of imatinib has been introduced to steer the drug impact toward clinically relevant imatinib targets (bcr-ABL, c-KIT, PDGFR kinases ${ }^{19}$ ), while suppressing off-target cross-reactivity against kinases such as LCK, whose inhibition may lead to harmful immunosuppressive effects. ${ }^{19}$ However, just like we may remove potentially harmful cross-reactivity by chemically modifying the parental scaffold guided by the wrapping filter, ${ }^{18}$ we could also incorporate new and therapeutically desirable cross-reactivity guided by a kinome-wide examination of IFEs.

By inputting the primary sequence, multiple sequence alignment, intrinsic disorder and secondary structure prediction, and pairwise residue coevolution, the IFEs for the 518 human kinases become accessible when adopting the DL platform described. As expected, the augmentation of the imatinib scaffold by the addition of a methyl group (WBZ 4 in Figure 3) would generally decrease cross-reactivity simply because fewer targets are typically able to accommodate a larger ligand as a result of steric hindrance. This is only partially true, however, as some targets are floppy enough to circumvent steric hindrance, especially those that may be susceptible to binding-induced 
folding. On the one hand, it was experimentally observed that WBZ 4 is not reactive against the imatinib target ABL kinase or against LCK, culprits of cardiotoxicity ${ }^{20}$ and immunosuppression, ${ }^{19}$ respectively. However, we found an exception to the steric hindrance rule in JNK1/2, which has no detectable affinity for imatinib but is a target for WBZ_4. ${ }^{9} \mathrm{JNK}$ stands out in the kinome-wide IFE inference because of its significant induced folding diversity in the targeted region and its susceptibility to getting wrapped by the extra methyl group not present in the imatinib scaffold, as shown in Figure 3. In fact, the nanomolar affinity of WBZ_4 against JNK1 has been experimentally documented, ${ }^{9}$ making this compound potentially impactful on ovarian cancer, where JNK inhibition has shown clinical relevance, at a variance with imatinib, which is not active in that therapeutic context. ${ }^{21}$

The DL-inferred IFE for JNK1 has two components described in Figure 3. An apo form (I), where the dehydron BHB M111N114 is not formed, and a holo form (II), which we reason must be the conformation adopted in the complex with WBZ_4. We arrived at that conclusion by docking WBZ_ 4 to JNK according to the structural alignment of JNK with the cKIT/imatinib complex reported in PDB.1T46 and noticing that WBZ_4 binding induces the BHB M111-N114 by contributing exogenously to its wrapping with the extra methyl that is not present in imatinib (Figure 3). Thus, the induced folding enabling the exogenous protection and, hence, stabilization of the induced M111-N114 BHB is responsible for the new crossreactivity that gets turned on by tuning the parental chemical scaffold.

This example illustrates the use of the DL platform to steer the reworking of a drug in order to redirect its impact toward a different therapeutic context. ${ }^{21}$

\section{DISCUSSION}

Rational drug design faces difficulties, and it is felt it may not be living up to the expectations. This is mostly because the target proteins are usually not fixed targets: they structurally adapt to the ligand in ways that have been very hard to predict. That brings us to the drug-induced folding problem that is squarely addressed in this work aimed at fueling pharmaceutical discovery within an AI-empowered platform.

We proposed and delineated a deep learning (DL) platform to teach drugs to target proteins. The DL system is geared at steering drug design to induce targetable binding-competent protein conformations. The control of induced folding by a drug or ligand requires a DL platform that, at a variance with protein folding predictors, integrates signals for the structural disorder to predict conformations of floppy regions that rely on purposely designed binding partnerships to maintain structural integrity. The conformational diversity of such regions begets an induced folding ensemble (IFE) from which the targetable conformation gets selected by DL-instructed drug design, enabling a therapeutically significant drug-target complexation.

In the preliminary testing presented of the AI-empowered discovery platform, we have dealt with fairly localized druginduced folding. Cases with extensive windows of intrinsic disorder susceptible to structural adaptation may prove more challenging, given the limited size of PDB-drawn training sets. Future work will assess the critical size of the induced folding region beyond which AI-directed drug design may require complementary training resources, possibly drawn from highlevel coevolution patterning.

\section{AUTHOR INFORMATION}

\section{Corresponding Author}

Ariel Fernández - CONICET, National Research Council, Buenos Aires 1033, Argentina; INQUISUR-CONICET-UNS, Bahía Blanca 8000, Argentina; Daruma Institute for AI in Pharmaceutical Research, AF Innovation GmbH, WinstonSalem, North Carolina 27106, United States; (1) orcid.org/ 0000-0002-5102-4294; Phone: 1-336-354-8315;

Email: ariel@afinnovation.com

Complete contact information is available at:

https://pubs.acs.org/10.1021/acs.molpharmaceut.0c00470

\section{Funding}

This research was supported in part through an unrestricted grant from Eli Lilly and Company, where the grantor agent was the former VP for information technology, Sangtae Kim.

Notes

The author declares no competing financial interest.

\section{REFERENCES}

(1) Senior, A. W.; Evans, R.; Jumper, J.; Kirkpatrick, J.; Sifre, L.; Green, T.; Qin, C.; Žídek, A.; Nelson, A. W.; Bridgland, A.; Penedones, H.; Petersen, S.; Simonyan, K.; Crossan, S.; Kohli, P.; Jones, D. T.; Silver, D.; Kavukcuoglu, K.; Hassabis, D. Improved protein structure prediction using potentials from deep learning. Nature 2020, 577, 706-710.

(2) Dill, K. A.; MacCallum, J. L. The protein folding problem, 50 years on. Science 2012, 338, 1042-1046.

(3) Jones, D. T.; Kandathil, S. M. High precision in protein contact prediction using fully convolutional neural networks and minimal sequence feature. Bioinformatics 2018, 34, 3308-3315.

(4) Jones, D. T.; Singh, T.; Kosciolek, T.; Tetchner, S. MetaPSICOV: combining coevolution methods for accurate prediction of contacts and long range hydrogen bonding in proteins. Bioinformatics 2015, 31, 999-1006.

(5) Yu, F.; Koltun, V. Multi-Scale Context Aggregation by Dilated Convolutions. 2016, arXiv:1511.07122.

(6) Chen, J. P.; Liang, H.; Fernández, A. Protein structure protection commits gene expression patterns. Genome Biol. 2008, 9, R107.

(7) Pietrosemoli, N.; Crespo, A.; Fernández, A. Dehydration Propensity of Order-Disorder Intermediate Regions in Soluble Proteins. J. Proteome Res. 2007, 6, 3519-3526.

(8) Ovchinnikov, V.; Louveau, J. E.; Barton, J. P.; Karplus, M.; Chakraborty, A. K. Role of framework mutations and antibody flexibility in the evolution of broadly neutralizing antibodies. eLife 2018, 7, e33038.

(9) Fernández, A.; Bazan, S.; Chen, J. Taming the induced folding of drug-targeted kinases. Trends Pharmacol. Sci. 2009, 30, 66-71.

(10) Schopf, F. H.; Biebl, M. M.; Buchner, J. The HSP90 chaperone machinery. Nat. Rev. Mol. Cell Biol. 2017, 18, 345-360.

(11) Plaxco, K. W.; Simons, K. T.; Baker, D. Contact order, transition state placement and the refolding rates of single domain proteins. J. Mol. Biol. 1998, 277, 985-994.

(12) Fernández, A.; Scheraga, H. A. Insufficiently dehydrated hydrogen bonds as determinants of protein interactions. Proc. Natl. Acad. Sci. U. S. A. 2002, 100, 113-118.

(13) Fernández, A. Physics at the Biomolecular Interface: Fundamentals for Molecular Targeted Therapy; Springer International Publishing: Switzerland, 2016.

(14) Fernández, A. Artificial Intelligence Steering Molecular Therapy in the Absence of Information on Target Structure and Regulation. J. Chem. Inf. Model. 2020, 60, 460-466.

(15) Obradovic, Z.; Peng, K.; Vucetic, S.; Radivojac, P.; Brown, C. J.; Dunker, A. K. Predicting Intrinsic Disorder from Amino Acid Sequence. Proteins: Struct., Funct., Genet. 2003, 53, 566-572. 
(16) Oldfield, C. J.; Dunker, A. K. Intrinsically Disordered Proteins and Intrinsically Disordered Protein Regions. Annu. Rev. Biochem. 2014, 83, 553-584.

(17) Rampasek, L.; Goldenberg, A. TensorFlow: Biology's Gateway to Deep Learning. Cell Sys. 2016, 2, 12-14.

(18) Druker, B. J.; Guilhot, F.; O'Brien, S. G.; Gathmann, I.; Kantarjian, H.; Gattermann, N.; Deininger, M. W.N.; Silver, R. T.; Goldman, J. M.; Stone, R. M.; Cervantes, F.; Hochhaus, A.; Powell, B. L.; Gabrilove, J. L.; Rousselot, P.; Reiffers, J.; Cornelissen, J. J.; Hughes, T.; Agis, H.; Fischer, T.; Verhoef, G.; Shepherd, J.; Saglio, G.; Gratwohl, A.; Nielsen, J. L.; Radich, J. P.; Simonsson, B.; Taylor, K.; Baccarani, M.; So, C.; Letvak, L.; Larson, R. A. Five-year follow-up of patients receiving imatinib for chronic myeloid leukemia. N. Engl. J. Med. 2006, 355, 2408-2417.

(19) Fernández, A.; Scott, L. R. Advanced Modeling Reconciles Counterintuitive Decisions in Lead Optimization. Trends Biotechnol. 2017, 35, 490-497.

(20) Kerkelä, R.; Grazette, L.; Yacobi, R.; Iliescu, C.; Patten, R.; Beahm, C.; Walters, B.; Shevtsov, S.; Pesant, S.; Clubb, F. J.; Rosenzweig, A.; Salomon, R. N.; Van Etten, R. A.; Alroy, J.; Durand, J. B.; Force, T. Cardiotoxicity of the cancer therapeutic agent imatinib mesylate. Nat. Med. 2006, 12, 908-916.

(21) Vivas-Mejia, P.; Benito, J. M.; Fernandez, A.; Han, H. D.; Mangala, L.; Rodriguez-Aguayo, C.; Chavez-Reyes, A.; Lin, Y. G.; Carey, M. S.; Nick, A. M.; Stone, R. L.; Kim, H. S.; Claret, F. X.; Bornmann, W.; Hennessy, B. T.; Sanguino, A.; Peng, Z.; Sood, A. K.; Lopez-Berestein, G. c-Jun-NH2-kinase-1 inhibition leads to antitumor activity in ovarian cancer. Clin. Cancer Res. 2010, 16, 184-194. 